\title{
Motion model for describing the quantity of air in droplets through changing the structure of air induction nozzle
}

\author{
Jun $\mathrm{Hu}^{1,2,3^{*}}$, Changxi Liu ${ }^{1,2,3}$, Zhichong Wang ${ }^{4}$, Yufei Li ${ }^{1,2,3}$, Jianli Song ${ }^{4}$, \\ Yajia Liu ${ }^{4}$ Xin $\mathrm{Chu}^{1,2,3}$ \\ (1. College of Engineering, Heilongjiang Bayi Agricultural University, Daqing 163319, Heilongjiang, China; \\ 2. Heilongjiang Provincial Key Laboratory of Intelligent Agricultural Machinery Equipment, Daqing 163319, Heilongjiang, China; \\ 3. Heilongjiang Province Conservation Tillage Engineering Technology Research Center, Daqing 163319, Heilongjiang, China; \\ 4. College of Science, China Agriculture University, Beijing 100193, China)
}

\begin{abstract}
Air induction nozzles possess good anti-drift performance, the throat and orifice sizes of the nozzles are the main design parameters that affecting atomization. Therefore, Venturi tube nozzles and conventional flat fan nozzles were assembled together to investigate the flow rate, droplet size, the quantity of air in droplets affected by a single design parameter of nozzles with applying high speed camera and Spraytec laser diffraction system. The results showed that: the flow rate of the air induction nozzle depended only on the throat size of Venturi tube and pressure, and it was proportional to the throat size of Venturi tube at the same pressure; The flat fan nozzle's orifice size and Venturi tube size significantly affected volume median diameter of droplets, which generally increased after adding surfactant; A new model was established after optimizing classical equation for calculating the percentage of intake air in droplets and studying the effects of throat and orifice size of air induction nozzles on spray characteristics. By variance analysis, it was verified that the new model of quantity of air in droplets produced by all connected nozzles was correct. The calculation showed that the bubbles sizes ranged at 200-900 $\mu \mathrm{m}$ and were in proportion to the droplet size with the percentage of intake air of $10 \%$ to $90 \%$. Contrast to the change of volume median diameter and droplet velocity, the existence of intake air could influence their change degree to some extent.
\end{abstract}

Keywords: throat orifice, spray characteristic, model of droplet motion, air induction nozzle DOI: $10.25165 /$ j.ijabe.20211405.5513

Citation: Hu J, Liu C X, Wang Z C, Li Y F, Song J L, Liu Y J, et al. Motion model for describing the quantity of air in droplets through changing the structure of air induction nozzle. Int J Agric \& Biol Eng, 2021; 14(5): 35-40.

\section{Introduction}

Air induction nozzles, generating negative pressure resulting to intake air when the high velocity fluid cross the Venturi tube and forming bubbly two-phase flow in a small space contributing to the atomization, are one key part to reduce drift potential ${ }^{[1]}$, so they can improve utilization of pesticides significantly ${ }^{[2-4]}$. Recently, beautiful countryside plan in China is gradually performed, and the hazards due to pesticide drift are taken more and more attentions, thus air induction nozzles with better anti-drift potential will be applied widely.

Atomization of pesticide, a heterogeneous phase, instant process with small size, wide range and a large number of droplets,

Received date: 2019-11-08 Accepted date: 2021-01-14

Biographies: Changxi Liu, PhD, Researcher, research interests: agricultural machine engineering. Email: 504924356@qq.com; Zhichong Wang, PhD, Researcher, research interests: pesticide science. Email: wzc91729@ hotmail.com; Yufei Li, MS, Researcher, research interests: agricultural machine engineering, Email:1637651412@qq.com; Jianli Song, PhD, Associate Professor, research interests: agricultural machine and spraying technology, Email: songjianli170@163.com; Yajia Liu, PhD, Associate Professor, research interests: pesticide science, Email: liuyajia@cau.edu.cn; Xin Chu, MS, Researcher, research interests: agricultural machine engineering, Email: 897242808@qq.com.

*Corresponding authors: Jun $\mathrm{Hu}, \mathrm{PhD}$, Professor, research interests: agricultural machine engineering. College of Engineering, Heilongjiang Bayi Agricultural University, No. 2 Xinyang Road, Longfeng District, Daqing 163319, China. Tel: +86-459-6819214, Email: gcxykj@126.com. directly determined pesticide deposition and $\operatorname{drift}^{[5]}$. Its characteristics reflect on droplet size and velocity, structure of spraying sheet, structure of droplet and so on ${ }^{[6-13]}$. These studies supplied a great deal of theoretical basis for controlling droplet parameters and could interpret the relationship between characteristics of droplet and liquid drift and deposition at a certain extent. Among them, content of air in droplet produced by air induction nozzles was especially studied to understand how design parameters affect spray characteristics and its anti-drift performance. Combellack et al. ${ }^{[14]}$ and Dorr et al. ${ }^{[15]}$ tested spray liquid density to characterize amount of gas contained in droplet. Ellis et al. ${ }^{[16]}$ studied the effect of structural characteristics of jet nozzle on spray characteristics and droplet density, calculated the content of bubbles according to equation. Meanwhile, its experimental results fit test values better. But it is difficult to explain nozzles performance by changing single variable due to their fixed Venturi tube's diameter and orifice size of integration nozzles.

Agratop Company produced a series of air induction nozzles with separable Venturi nozzle and standard interface. In order to understand more information about anti-drift characteristics of air induction nozzles, this study assembled flat fan nozzle from Lechler company with Agratop company's Venturi tube to study droplet size spectrum, droplet velocity by changing single variable, and established new motion equation through mathematical and physical methods to calculate the quantity of air in droplet. This test was a reference for the accurate calculation of the quantity of 
air in droplets for the future. In addition, according to the flow rate, droplet size, the quantity of air in droplets required by the actual operation situation, the air induction nozzles with anti-drift characteristics can be designed and used to further reduce the possibility of environmental pollution caused by the drift of fog drops.

\section{Materials and methods}

\subsection{Test materials and contents}

Before the test, the combination of Turbo Drop Venturi tube (Agrotop, Germany), and flat fan nozzles (Lechler, Germany) was screened as listed in Table 1 (under the condition of $0.2,0.3$, $0.4 \mathrm{MPa}$, respectively).

Table 1 Slit width data of Venturi tube and fan nozzle that meet the test requirements

\begin{tabular}{ccccccc}
\hline & ST110-02 & ST110-03 & ST110-04 & ST110-05 & TD02 & TD03 \\
\hline $\begin{array}{c}\text { Slit width } \\
/ \mathrm{mm}\end{array}$ & 0.84 & 0.99 & 1.20 & 1.40 & 1.33 & 1.77 \\
\hline
\end{tabular}

When TD-03 Venturi tube connected with ST110-03 and ST110-04 nozzles, the liquid shed from nozzles, and TD-04 and TD-05 Venturi tube connected any ST series nozzles occurred leakage. Thereby only TD02-ST11003, TD02-ST11004, TD02-ST11005, TD02-ST11006, TD03-ST11005, TD03-ST11006 connected nozzles were tested in this study (as shown in Figure 1). Therefore, the orifice size of ST11003, ST11004, ST11005 and ST11006 fan nozzle and the throat size of TD-02 and TD-03 Venturi tube that meet the test requirements were given, as shown in Table 1 (Photos of Venturi tube and fan nozzle' diameter and ruler were taken at same magnification times and focal length).

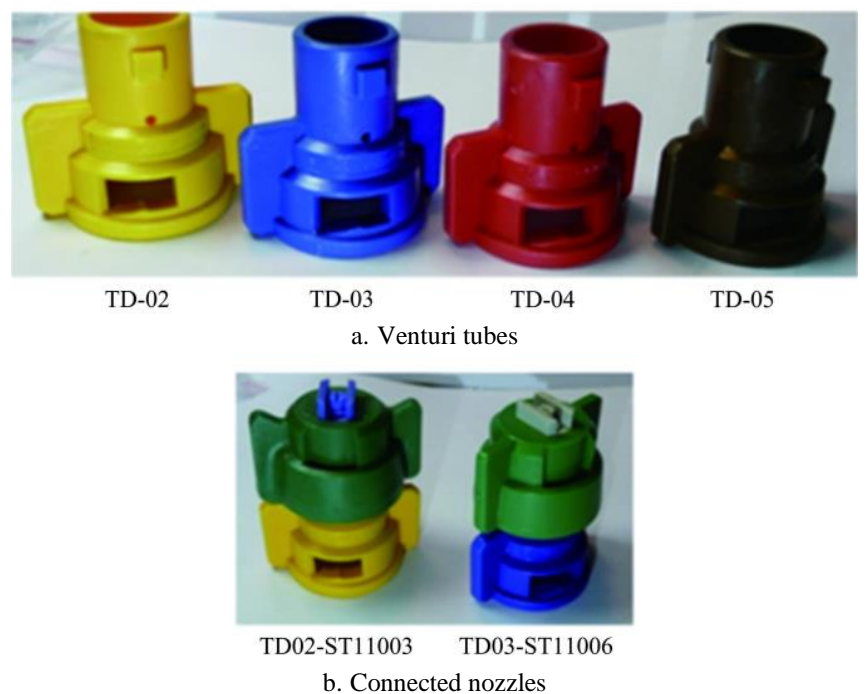

Figure 1 Venturi tubes (TD-02, TD-03, TD-04, TD-05) and connected nozzles (TD02-ST11003, TD03-ST11006)

In the test, water and $0.05 \%$ Silwet 408 were respectively used as spray fluid. The test content included flow rate, volume median diameter, droplet sizes and velocity of droplet (all tests were repeated in triplicate). The observation content was the droplet structure, and the calculation content was the percentage of air content percent.

\subsection{Test equipment and methods}

\subsubsection{Measurements of flow rate}

Flow rates of nozzles were obtained from nozzle tester S001 (AAMS-Salvarani, Belgium). When it displayed stable data, the data of flow rate was recorded. The spray liquid was water and $0.05 \%$ Silwet 408 individually. All tests were repeated 3 times.
2.2.2 Measurements of volume median diameter and observation of droplet structure

Connected nozzles were tested respectively at $0.4 \mathrm{MPa}$ by Spraytec laser diffraction system (Malvern, England). The test point was $50 \mathrm{~cm}$ away from nozzle in the central line of nozzle. The laser beam was perpendicular to the spray fan through the test points to gather the data of volume median diameter. At the same test point, droplets were collected by petri dish filled with silicone oil. Then structure of droplets was immediately observed with Digital microscope (Dino-lite, China). All tests were repeated in triplicate.

2.2.3 Measurements of droplet sizes and velocity

High speed camera (Lightning RTDTM (PLUS), DRS, American) was applied to measure the droplet size and velocity at 0.4 MPa with water and $0.05 \%$ Silwet 408 individually for all connected nozzles. The shutter speed of high speed camera was $1 / 10000 \mathrm{~s}$, the frame rate was $5000 \mathrm{fps}$. Spray sheet was parallel to lens of the high speed camera. Every test was repeated 3 times. Ellis et al. ${ }^{[16]}$ mentioned there was unsteady state in the progress of spraying, and expected that mainly affected by nozzle orifice size to a great extent. When spraying with TD03-ST11006, there was louder sound compared with other nozzles. In order to avoid the effect of air flow on spray, test point A was selected at $33 \mathrm{~cm}$ away from nozzle in vertical line of spray fan avoiding the effect of air flow; test point $\mathrm{B}$ was selected $55 \mathrm{~cm}$ away from nozzle in vertical line of spray fan.

A video containing the ruler at the same test point needed to be recorded before spray. By analyzing clear droplet in two images of adjacent frames, droplet size and velocity can be calculated with high speed camera soft.

\subsection{Calculation of air content percent}

Liu et al. ${ }^{[17]}$ established several kinds of more simple equations of motion according to air resistance. Its basic structure is:

$$
\frac{\mathrm{d} V}{\mathrm{~d} t}=g^{\prime}-k V
$$

where, $g^{\prime}$ is the gravitational acceleration, $\mathrm{m} / \mathrm{s}^{2} ; k$ is a constant; $V$ is the mean velocity of droplets, $\mathrm{m} / \mathrm{s}$; and $t$ is the time step, $\mathrm{s}$.

The parameters of $g^{\prime}, k$ and the powers of mean velocity are variable terms. The droplet motion equations were firstly built by changing the above variable terms to determine expression of $g^{\prime}, k$ and the powers of mean velocity.

According to Kelvin equation ${ }^{[18]}$, there are no bubbles in droplet when spraying water, so data of water droplet were used to establish the correct model. That is to say, the water density $\rho$ in the models is $1000 \mathrm{~kg} / \mathrm{m}^{3}$, the acceleration of gravity is $9.81 \mathrm{~m} / \mathrm{s}^{2}$, air density is $1.293 \mathrm{~kg} / \mathrm{m}^{3[19]}$, the viscosity of the air is $1.80 \times 10^{-5} \mathrm{~Pa} \cdot \mathrm{s}$ when relative humidity is $100 \%{ }^{[20]}$ in the equation. In order to avoid calculating large data, TD02-ST11003 and TD03-ST11006 under extreme circumstances were selected to build the new model.

Assuming that the changeable step of time was one hundred-thousandth and velocity index was fixed value, the variable values of acceleration, distance and velocity was obtained by progressive searching according to

$$
\begin{gathered}
V=V_{a}+a t \\
S=V_{a} t+\frac{1}{2} a t^{2} \\
a=\frac{\mathrm{d} V}{\mathrm{~d} t}
\end{gathered}
$$

where, $a$ is acceleration, $\mathrm{m} / \mathrm{s}^{2} ; V_{a}$ is the initial velocity of droplets, $\mathrm{m} / \mathrm{s} ; S$ is the distance of movement, $\mathrm{m}$. 
Applying IF function, the change of time would stop automatically when velocity was less than that of droplets at test point B. Supposing the change of distance as the target, and making actual changeable distance as the distance between point $\mathrm{A}$ and B, velocity index was calculated through goal seek.

The standard determining whether the model was suitable for spray droplets in this study was: variation coefficient of $e$ in velocity index for all the droplets separately produced by TD02-ST11003 or TD03-ST11006 were less than 10\%, and there were no significant differences between TD02-ST11003 and TD03-ST11006 at 95\% confidence interval, then the model was eligible.

In line with the above calculation progress of velocity index, the expressions of $g^{\prime}, k$ can be determined in sequence according to the above reliable standard.

Eventually, an innovation model for calculation was established. Supposing the change of distance as target, and making actual changeable distance as the distance between A point and B point, droplets density was calculated through goal seek according to the calculation progress of velocity index. Assuming that droplet is spherical, the equation for air content percentage is:

$$
\begin{gathered}
\rho=\frac{\rho_{l} V_{l}+\rho_{g} V_{g}}{V_{l}+V_{g}} \\
V_{g}=\frac{4}{3} \pi\left(\frac{D}{2}\right)^{2}-V_{l}
\end{gathered}
$$

where, $\rho$ is the density of liquid and air in droplet, $\mathrm{kg} / \mathrm{m}^{3} ; D$ is the diameter of droplet, $\mathrm{m} ; \rho_{l}$ is the density of liquid in droplet, $\mathrm{kg} / \mathrm{m}^{3}$; $\rho_{g}$ is the density of air in droplet, $\mathrm{kg} / \mathrm{m}^{3} ; V_{l}$ is the volume of liquid in droplet, $\mathrm{m}^{3}$; and $V_{g}$ the volume of air in droplet, $\mathrm{m}^{3}$.

\section{Results and analysis}

\subsection{Effects of throat and orifice size on flow rate}

When testing separate Venturi tube and flat fan nozzle under the same pressure with water, flow rate was proportional to crosssectional area in Figure 2a, agreeing with the Bernoulli equation.

After connecting Venturi tube and flat fan nozzle, flow rate of all connected nozzles increased in Figure $2 b$. When spraying $0.05 \%$ Silwet 408, TD02 and TD03 Venturi tubes were 1.14 times and 1.13 times more than that of spraying water. Whether spraying water or $0.05 \%$ Silwet 408 solution and which ST series nozzles that TD-02 and TD-03 connected with, the liquid flow rates were similar individually for TD02-ST series and TD03-ST series.
Flow rate depended only on the Venturi tube throat size, and the bigger throat size was, the more flow rate was. Meanwhile, flow rate gradually increased with the augment of pressure.



a. Venturi tube and flat fan nozzle

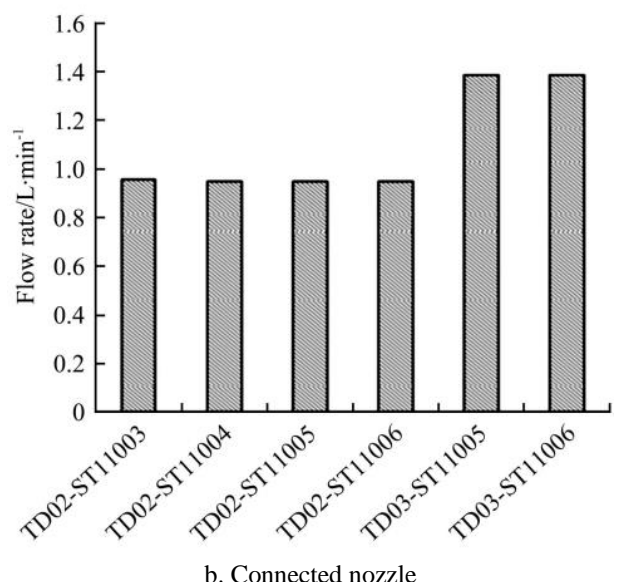

Figure 2 Flow rates of separate Venturi tube and flat fan nozzle (a) and connected nozzle (b)

3.2 Effect of throat and orifice size on volume median diameter of droplet and observation of droplet structure

It can be seen from Table 2, volume median diameter (VMD) of droplet increased with the augment of ST series nozzle' orifice size, and decreased with the increase of TD series Venturi tube' throat size for connected nozzles.

Volume media diameter of droplet without adjuvant increased was more than that with adding additives. It showed that air in droplet could slow down the change of droplets' VMD affected by nozzle structure, which agreed with that from Dorr et al. ${ }^{[15]}$

\begin{tabular}{|c|c|c|c|c|c|c|c|c|}
\hline & \multicolumn{4}{|c|}{ TD-02 } & \multicolumn{4}{|c|}{ TD-03 } \\
\hline & ST110-03 & ST110-04 & ST110-05 & ST110-06 & ST110-03 & ST110-04 & ST110-05 & ST110-06 \\
\hline Water & 212.5 & 239.2 & 375.3 & 507.3 & - & - & 243.8 & 357.4 \\
\hline $0.05 \%$ Silwet 408 & 250 & 318.2 & 395.4 & 487.4 & - & - & 307.6 & 385.5 \\
\hline
\end{tabular}

Table 2 Droplet VMD ( $\mu \mathrm{m})$ for all connected nozzles when spraying water or $0.05 \%$ Silwet 408

\subsection{Effect of adjuvant on droplet structure}

After observation, there was no bubble in droplet when spraying water in Figure 3a; but there were bubbles in droplet when spraying $0.05 \%$ Silwet 408 liquid in Figure 3b. That agreed with the result from Kelvin equation.

According to the above results, it was supposed that VMD of droplets was closely related to the structure of droplets.

\subsection{Calculation of droplet size and velocity}

MiDAs Player software was used to analyze the obtained video. Only clear droplets without disturbance at points A and B were selected to be analyzed. In statistics, when the movement direction of droplet was $10^{\circ}$ away from the vertical direction, this droplet was excluded. In principle, the droplets with the same particle size were not less than five for statistical analysis, so the total number of droplets with different sizes was not less than 60 for every tested nozzle. The range of analyzed droplets was 100 $900 \mu \mathrm{m}$.

Meanwhile, the distance between A and B was measured and recorded so long as changing nozzle and spray liquid. The results were listed in Table 3. 


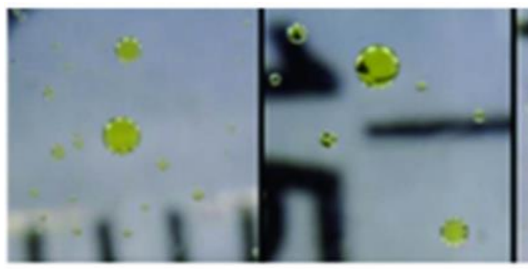

TD02-ST11003

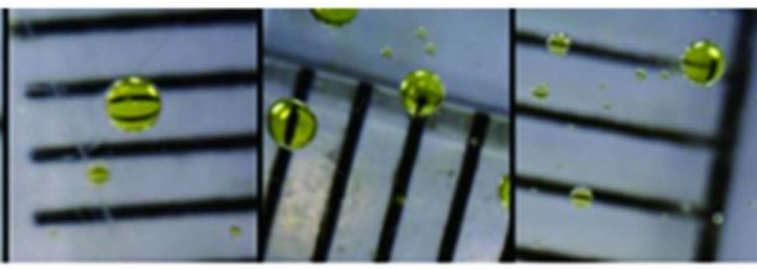

TD02-ST11005
TD02-ST11006 a. Water

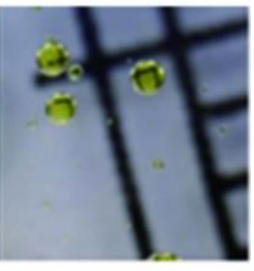

TD03-ST11006

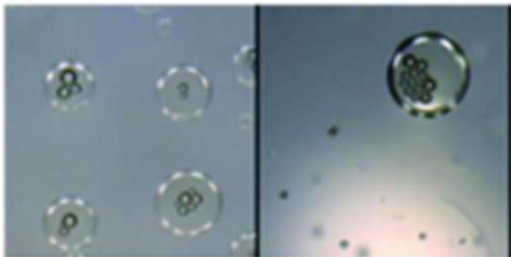

TD02-ST11003
TD02-ST11004

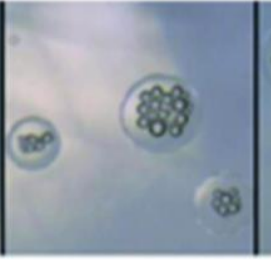

TD02-ST11005

\section{b. $0.05 \%$ Silwet 408}

TD03-ST11005

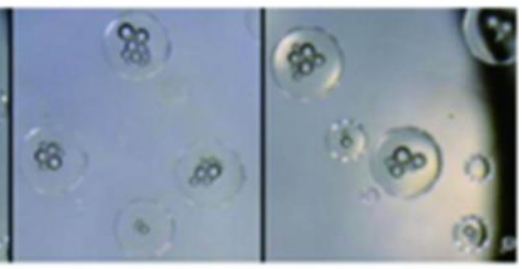

TD03-ST11005

TD03-ST11006

Figure 3 Structures of droplets produced by connected nozzles spraying water and $0.05 \%$ Silwet 408

Table 3 Distances between $A$ and $B$

\begin{tabular}{lc}
\hline & Distance between A and B/cm \\
\hline TD02-ST03 (water) & 29.25 \\
TD02-ST11003 (0.05\% Silwet 408) & 24.08 \\
TD02-ST11004 (0.05\% Silwet 408) & 24.73 \\
TD02-ST11005 (0.05\% Silwet 408) & 24.73 \\
TD02-ST11006 (0.05\% Silwet 408) & 27.69 \\
TD03-ST11005 (0.05\% Silwet 408) & 24.73 \\
TD03-ST11006 (water) & 29.25 \\
TD03-ST11006 (0.05\% Silwet 408) & 24.73 \\
\hline
\end{tabular}

From Figures 4 and 5, when the size of droplet was more 200 $\mu \mathrm{m}$, velocity of droplet increased gradually with the increase of droplet size regardless of nozzle orifice size and Venturi tube throat size. Whether the test point was A or B, for TD-02, TD-03 Venturi tube, the bigger nozzle orifice size was, the lower velocity for the same size droplet was. And the bigger venture tube throat size was, the higher velocity for the same size droplet was except TD02-ST11003 nozzle. Take TD-02 connected nozzles as an example, the difference of velocity between A and B decreased gradually with the increase of droplet size by comparing the slope of velocity curve for all connected nozzles.

From Ellis ${ }^{[16]}$, when the test point was $200 \mathrm{~mm}$ below nozzles, the result was consistent with above consequence, however when test point was $600 \mathrm{~mm}$ below the nozzles, they were not the same. This may be because that it was determined by the design parameters of nozzle.

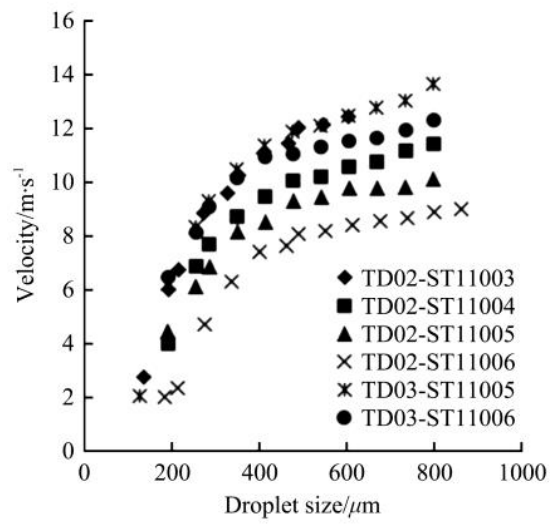

Figure 4 Trend of mean velocity of droplets produced by connected nozzles at test point $\mathrm{A}$

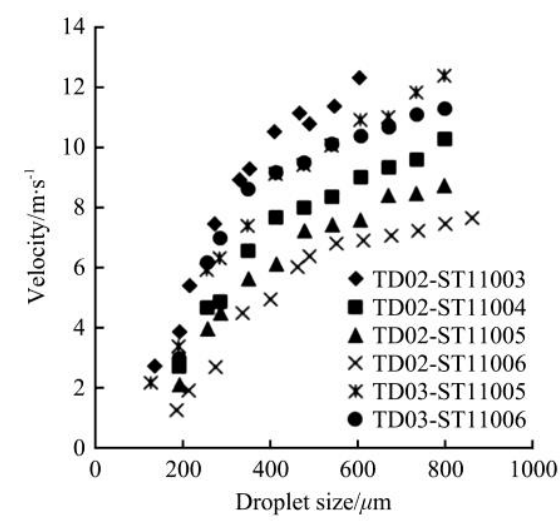

Figure 5 Trend of mean velocity of droplets produced by connected nozzles at test point $\mathrm{B}$

\subsection{Establishment of the new model}

Firstly, exported motion equations were examined to know whether it was proper to droplets in this study.

The results showed that coefficient variation of all models was greater than $10 \%$, and the maximum was up to $58.77 \%$. There were significant intra-group difference, and coefficient variation of TD02-ST11003 was greater than that of TD03-ST11006.

For the changeable coefficients in models, the basic optimization method is changing one parameter, remaining the other parameters, then calculating velocity index by goal seek and making its coefficient variation less than $10 \%$. But all optimized model had significant difference in $95 \%$ confidence interval. That's to say all optimized models were not suitable for droplet in this study.

So this study established a new model, and its basic structure is as follow:

$$
\frac{\mathrm{d} V}{\mathrm{~d} t}=g^{\prime}-k V^{e \cdot \ln (V)+b}
$$

where, $e$ and $b$ express unknown constant, the gravitational acceleration is given by

$$
g^{\prime}-\left[1-\frac{m^{\prime}}{m}\right] g=\left[1-\frac{\rho_{g}}{\rho_{l}}\right] g
$$

where, $m^{\prime}$ is the mass of air in droplet, $\mathrm{kg}$; and $m$ is the mass of liquid in droplet, $\mathrm{kg}$; and the $k$ is given by

$$
k=\frac{6 \pi \eta r}{m}=\frac{9 \eta}{2 r^{n} \rho_{l}}
$$


where, $\eta$ is the viscosity of air in droplet, $\mathrm{Pa} \cdot \mathrm{s} ; r$ is the radius of droplet, $\mathrm{m}$; and $n$ is the powers of $r$. And the coefficient of $k, n, e$ and $b$ are variable terms.

Assuming $b$ was zero, $e$ could be obtained through goal seek with changeable $n$. Then $n$ could be determined until the coefficient variation of $e$ was less than $10 \%$. With the above method, $b$ could be optimized. The optimal result is as followed:

$$
\frac{\mathrm{d} V}{\mathrm{~d} t}=g^{\prime}-k V^{e \cdot \ln (V)-1}
$$

where the express of $k$ is

$$
k=\frac{5 \eta}{r^{2.1} \rho_{l}}
$$

All the calculated values of $e$ are listed in Table 4 .

Table 4 Calculated values of $e$ in velocity index term and droplets of TD02-ST11003, TD03-ST11006

\begin{tabular}{ccc}
\hline & Droplet size/ $\mu \mathrm{m}$ & $e$ \\
\hline & 152 & 1.093 \\
214 & 1.205 \\
TD02-ST11003 & 338 & 1.265 \\
& 399 & 1.264 \\
& 489 & 1.091 \\
& 551 & 1.155 \\
& 613 & 1.149 \\
& 214 & 1.137 \\
\hline TD03-ST11006 & 276 & 1.172 \\
& 338 & 1.29 \\
& 399 & 1.137 \\
& 489 & 1.111 \\
& 551 & 1.138 \\
& 613 & 1.14 \\
675 & 1.104 \\
& 737 & 1.076 \\
& & 1.065 \\
\hline
\end{tabular}

According to sample features, $T$-test of independent sample was selected. From results, as the variance of all of $e$ were equal ( $F=0.428, p=0.623>0.05$ ), the value of test statistic $T$ was 1.002 , and the associated probability was $0.332>0.05$, so there was no significant difference between these two groups data. Therefore, the innovation model was suitable for TD-ST series droplets.

The coefficient variation of $e$ was less than $6 \%$, and there was no significant difference for all droplets. Then, all of $e$ was averaged, and the model was determined:

$$
\frac{\mathrm{d} V}{\mathrm{~d} t}=g^{\prime}-k V^{1.152 \cdot \ln (V)-1}
$$

where, $g^{\prime}=\left[1-\frac{m^{\prime}}{m}\right] g=\left[1-\frac{\rho_{\text {air }}}{\rho_{\text {water }}}\right] g, k=\frac{5 \eta}{r^{2.1} \rho_{\text {water }}}$.

\subsection{Air content percentage of droplet}

According to equation, the air content percentage of droplet was calculated. From Figure 6, there were bubbles in droplet after adding $0.05 \%$ Silwet 408 into spraying liquid, and that verified the phenomenon got from silicone oil. This also could well explain why the size of droplets increased after adding the Silwet 408 .

Contrast to the change of droplet' VMD, air content percentage of droplet had increased trend with increase of droplet size.

Contrast to the change degree of velocity, the more air content percentage of droplet was, the less change degree of velocity between A and B was. According to Equation (12), the more air content percentage of droplets was, the smaller acceleration gravity was. So it well explained the phenomenon that change degree of velocity for droplet moved from A to B decreased with the increase of droplet air content percentage.

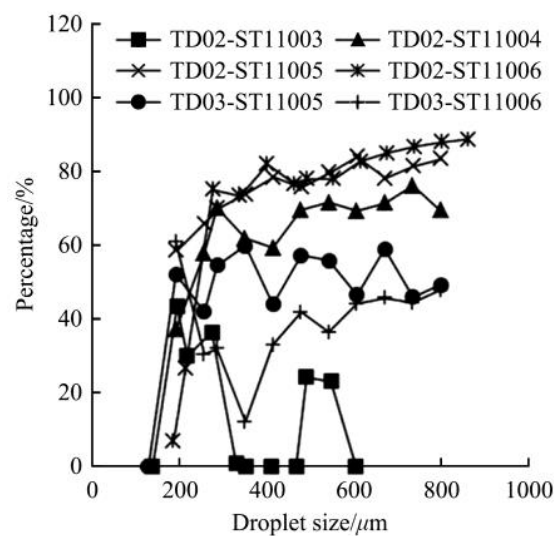

Figure 6 Effects of droplet size on percentage of intake air

When the nozzles were connected to the TD-02 Venturi tube, the quantity of air increased with the increase of the final nozzles orifice. But when the nozzles connected to the TD-03 Venturi tube, the quantity of air increased with the decrease of final nozzles orifice. When droplet size was more than $276 \mu \mathrm{m}$, air content percentage of droplets produced by TD-02 connected nozzles was higher than that produced by TD-03 connected nozzles except TD02- ST11003 and TD03-ST11003.

Analysis of data from TD02-ST11003 and TD03-ST11006 were as followed:

For TD02-ST11003 nozzle, small droplet make up a large proportion of droplet spectrum, so it was easy to be affected by measuring error and spray conditions.

For TD03-ST11006 nozzle, the flow rate was larger compared with other nozzles which may make the intake air erupt with fast flow and not be involved into droplets.

\section{Discussion}

Compared with the integral nozzle, this study accurately illuminated design parameters how to influence flow rate, VMD of droplet and velocity, which provide significant new conclusions for atomizing theory. Combination of change trend of VMD and velocity of droplet, it was found that VMD of droplet and air constant could interact on each other, and change degree of velocity was affected to a large extent.

According to the basic theory of motion equation, this study built a new model through optimization of variable and supply a new method to analyze density of droplet. This equation can quantify the constant of air in droplet, and clearly show design parameters how to affect the change of air constant in droplets. Ellis et al. ${ }^{[16]}$ reported their investigations that air intake would increase with incensement of throat size, orifice size or VMD of droplet, whereas droplet density was decreased. But the results in this paper are not exactly the same. The innovative model was suitable for all kinds of droplet produced by TD-ST combined air induction nozzles and could calculate the air content well. So it could be an important theory basis for figuring out the relationship between air intake and air constant in droplet.

\section{Conclusions}

In order to study the effect of single variable of air induction nozzles structure on pesticide atomization, Turbo Drop Venturi 
tube and conventional flat fan nozzle were assembled to investigate property of atomization. The following conclusions are obtained:

1) The flow rate of the air induction nozzles depended only on the diameter of the orifice size and was proportional to the square of the diameter. The VMD of the droplet was mainly affected by the nozzle size and was linear with it. After the $0.05 \%$ Silwet 408 was added, the droplets produced by the air induction nozzles did not contain bubbles, and the volume median particle size was generally increased.

2) By statistics, a new motion model for all droplets was successfully built to calculate the percentage of air in droplet. It was proved that the quantity of air in droplets after adding surfactant to air induction nozzles can be calculated by a certain mathematical model, and it was not irregular. The investigations can clearly show relationship between droplet size and content of intake air, and usefully explain change degree of droplet velocity to a certain extent.

3) Though it was found that obtained results were not totally agreed with that got from integrated nozzles, all results clearly stated theory and property of atomization about air induction nozzles any further. This research provided a certain reference for the design and use of air induction nozzles with anti-drift characteristics in the future to further reduce the possibility of droplets drift on environmental pollution.

\section{Acknowledgements}

We acknowledge that this work was financially supported by National Key Research and Development Program (No.2016YFD0200706), Heilongjiang Bayi Agricultural University Academic Achievement Introduction Project (No.XDB2013-08), Natural Science Outstanding Youth Project of Heilongjiang Province of China (No.YQ2019E032) and University Level Key Project of Heilongjiang Bayi Agricultural University (No.XA2015-01). We special thanks to the support of National Soybean Industry Technology System Post Expert Foundation of China (No.CARS-04-01A) funded by China Agriculture Research System of MOF and MARA.

\section{[References]}

[1] Zhan Q, Zeng A J, He X Q, Song J L, Zhou J Z. Effervescent atomizers and the prospect in pesticide application. Science Paper Online, 2007; 2(2): 134-139. (in Chinese)

[2] Derksen R C, Zhu H, Fox R D, Brazee R D, Krause C R. Coverage and drift produced by air induction and conventional hydraulic nozzles used for orchard applications. Transactions of the ASABE, 2007; 50(5): 1493-1501.
[3] Peter H S, Lynette B, Christy S, Helmut S, Nader S. Flat fan and air induction nozzles affect soybean herbicide efficacy. Weed Biology and Management, 2008; 8(1): 31-38.

[4] McArtney S J, Obermiller J D. Comparative performance of air-induction and conventional nozzles on an axial fan sprayer in medium density apple orchard. Herb Technology, 2008; 18(3): 365-371.

[5] Andrzej G, Witold B, Paulina M. Spray coverage in potatoes with low drift and air-induction nozzles. Journal of Plant Protection Research, 2005; 45(1): 17-23.

[6] Lv X L, He X K, Song J L, Zeng A J, Andreas H. Analysis of spray process produced by agriculture flat fan nozzles. Transactions of the CSAE, 2007; 16(9): 95-100. (in Chinese)

[7] Miller P C H, Ellis M C B, Tuck C R. Entrained air and droplet velocities produced by agricultural flat fan nozzle. Atomization and Sprays, 1996; 6(6): 693-707.

[8] Nuyttens D, Baetens K, Schampheleire M D, Sonck B. Effect of nozzle type, size and pressure on spray droplet characteristics. Biosystems Engineering, 2007; 97(3): 333-345.

[9] Song J L, Liu Y J, Zhang J, He X K, Zeng A J, Andreas H. Drift mechanism of flat fan nozzle. Transactions of the CSAM, 2011; 42(6): 63-69. (in Chinese)

[10] Knewitz H, Weisser P, Koch H. Drift-reducing spray application in orchards and biological efficacy of pesticides. Aspects of Applied Biology, 2002; 66(1): 231-236.

[11] Jones E J, Hanks J E, Wills G D. Effect of different nozzle types on drift and efficacy of roundup ultra. Office of Agricultural Communication, Division of Agriculture, Forestry, and Veterinary Medicine, Mississippi State University, 2002; 53(3): 11-15.

[12] Zhang J, Song J L, He X K, Zeng A J, Liu Y J. Droplets Movement Characteristics in Atomization Process of Flat Fan Nozzle. Transactions of the CSAM, 2011; 42(4): 66-70. (in Chinese)

[13] Zhao H, Song J L, Zeng A J, He X K. Correlations between dynamic surface tension and droplet diameter. Transactions of the CSAM, 2009; 40(8): 74-79. (in Chinese)

[14] Combellack J H, Western N M, Richardson R G. A comparison of the drift potential of a novel twin fluid nozzle with conventional low volume flat fan nozzles when using a range of adjuvants. Crop Protection, 1996; 15(2): $147-152$

[15] Dorr G J, Hewitt A J, Adkins S W, Hanan J, Zhang H, Noller B. A comparison of initial spray characteristics produced by agricultural Nozzles. Crop protection, 2013; 53: 109-117.

[16] Ellis M C B, Swan T, Miller P C H, Waddelow S, Bradley A, Tuck C R. $\mathrm{PM}$ - power and machinery: Design factors affecting spray characteristics and drift performance of air induction nozzles. Biosystems Engineering, 2002; 82(3): 289-296.

[17] Liu J J, Zhou X Z. Common discussion of the terminal velocity of the falling raindrops. Physics and Engineering, 2010; 20(5): 17-19. (in Chinese)

[18] Fu X C, Shen W X, Yao T Y, Hou W H. Physical chemistry. Beijing: High Education Press, 2006; 324p.

[19] Wen R Y, Yan S Q, Jiang H, Zhai M L. Basics of chemical engineering. Beijing: The Peking University Publishing House, 2002; 259p.

[20] Tsilingiris P T. Thermophysical and transport properties of humid air at temperature range between 0 and $100^{\circ} \mathrm{C}$. Energy Conversion and Management, 2008; 49(5): 1098-1110. 